On the origin of magnetic anisotropy in two dimensional Crl\$_3\$

This content has been downloaded from IOPscience. Please scroll down to see the full text.

Download details:

IP Address: 193.145.230.254

This content was downloaded on 07/06/2017 at 12:47

Manuscript version: Accepted Manuscript

Lado et al

To cite this article before publication: Lado et al, 2017, 2D Mater., at press:

https://doi.org/10.1088/2053-1583/aa75ed

This Accepted Manuscript is: @ 2017 IOP Publishing Ltd

During the embargo period (the 12 month period from the publication of the Version of Record of this article), the Accepted Manuscript is fully protected by copyright and cannot be reused or reposted elsewhere.

As the Version of Record of this article is going to be / has been published on a subscription basis, this Accepted Manuscript is available for reuse under a CC BY-NC-ND 3.0 licence after the 12 month embargo period.

After the embargo period, everyone is permitted to copy and redistribute this article for non-commercial purposes only, provided that they adhere to all the terms of the licence https://creativecommons.org/licences/by-nc-nd/3.0

Although reasonable endeavours have been taken to obtain all necessary permissions from third parties to include their copyrighted content within this article, their full citation and copyright line may not be present in this Accepted Manuscript version. Before using any content from this article, please refer to the Version of Record on IOPscience once published for full citation and copyright details, as permission will likely be required. All third party content is fully copyright protected, unless specifically stated otherwise in the figure caption in the Version of Record.

When available, you can view the Version of Record for this article at:

http://iopscience.iop.org/article/10.1088/2053-1583/aa75ed 


\title{
On the origin of magnetic anisotropy in two dimensional $\mathrm{CrI}_{3}$
}

\section{J. L. Lado ${ }^{1}$ and J. Fernández-Rossier ${ }^{1,2}$}

${ }^{1}$ QuantaLab International Iberian Nanotechnology Laboratory (INL), Av. Mestre José Veiga, 4715-330 Braga, Portugal

${ }^{2}$ Departamento de Física Aplicada, Universidad de Alicante, 03690 Spain

\begin{abstract}
.
The observation of ferromagnetic order in a monolayer of $\mathrm{CrI}_{3}$ has been recently reported, with a Curie temperature of 45 Kelyin and off-plane easy axis. Here we study the origin of magnetic anisotropy, a necessary ingredient to have magnetic order in two dimensions, combining two levels of modeling, density functional calculations and spin model Hamiltonians. We find two different contributions to the magnetic anisotropy of the material, favoring off-plane magnetization and opening a gap in the spin wave spectrum. First, ferromagnetic super-exchange across the $\simeq 90$ degree Cr-I-Cr bonds, are anisotropic, due to the spin orbit interaction of the ligand I atoms. Second, a much smaller contribution that comes from the single ion anisotropy of the $S=3 / 2 \mathrm{Cr}$ atom. Our results permit to establish the XXZ Hamiltonian, with a very small single ion easy axis anisotropy, as the adequate spin model for this system. Using spin wave theory we estimate the Curie temperature and we highlight the essential role played by the gap that magnetic anisotropy induces on the magnon spectrum.
\end{abstract}


On the origin of magnetic anisotropy in two dimensional $\mathrm{CrI}_{3}$

\section{Introduction}

The recent reports of ferromagnetic order in two different two dimensional crystals, $[1,2]$ $\mathrm{Cr}_{2} \mathrm{Ge}_{2} \mathrm{Te}_{6}$ and $\mathrm{CrI}_{3}$, together with the report of antiferromagnetic order[3, 4] in FePS a few months earlier, mark the beginning of a new chapter in the remarkable field of two dimensional materials. These discoveries extend significantly the list of electronically ordered two dimensional crystals, that included already superconductors, $[5,6]$ charge density waves materials[7] and ferroelectrics.[8] In addition, there is an increasing amount of computational studies predicting magnetic order in large variety of two dimensional materials, such as $\mathrm{VS}_{2}$ and $\mathrm{VSe}_{2},[9] \mathrm{K}_{2} \mathrm{CuF}_{4},[10]$ and the family of $\mathrm{MPX}_{3}$, with $\mathrm{M}$ the $3 \mathrm{~d}$ transition metals and $\mathrm{X}$ a group VI atom.[11] The integration of magnetically ordered 2D crystals in Van der Waals heterostructures[12] opens a vast field of possibilities for new physical phenomena and new device concepts, and is already starting to be explored experimentally.[13]

Mermin and Wagner demonstrated the absence of long range magnetic order in spinrotational invariant systems with short range exchange interactions.[14] Therefore, the observation of long range magnetic order in two dimensional insulating materials stresses the importance of a quantitative microscopic understanding of magnetic anisotropy in these systems. The breaking of spin rotational invariance can be due to three mechanisms, dipolar interactions, single ion anisotropy and anisotropy of the exchange interactions. In the case of very strong single ion anisotropy, a description in terms of the Ising model could be possible, which automatically entails a magnetically ordered phase phase at finite temperature, as predicted by Onsager in his remarkable paper.[15] However, large single ion anisotropies are normally associated to partially unquenched orbital moment of the magnetic ion, which only happens for specific oxidation states and low symmetry crystal environments, most notably in surfaces[16] or for rare earth atoms.[17]

$\mathrm{CrI}_{3}$ is a layered transition metal compound known to order ferromagnetically, in bulk, at $T_{c}=61$ Kelvin.[18, 19] Ferromagnetic order has been shown to persist in mechanically exfoliated monolayers of $\mathrm{CrI}_{3}$, with a Curie Temperature of $T_{c}=45$ Kelvin, as determined by magneto-optical measurements.[2] In this work we model magnetic anisotropy in a monolayer of $\mathrm{CrI}_{3}$. Since dipolar interactions favor in-plane anisotropy, we focus on the study of/both single ion anisotropy and exchange anisotropies. To do that, we first model the system with relativistic all electron density functional theory (DFT) calculations that include spin orbit interactions, essential to account for magnetic anisotropy. Our calculations permit to build an effective spin model with three energy scales, the isotropic and anisotropic Cr-Cr exchange couplings, $J$ the anisotropic exchange $\lambda$, and the single ion anisotropy $D$. As we show below, $J$ and $\lambda$ are non zero, whereas the single ion anisotropy $D$ is negligible.

Both experimental results [19, 2] and DFT calculations $[20,21]$ show that $\mathrm{CrI}_{3}$ is an semiconducting material with a the band-gap of $1.2 \mathrm{eV}$.[22] In a single layer of $\mathrm{CrI}_{3}$, the plane of $\mathrm{Cr}$ atoms form a honeycomb lattice and is sandwiched between two 
(a)

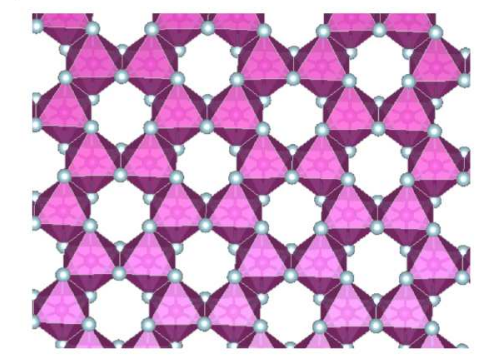

(b)

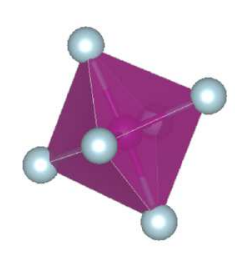

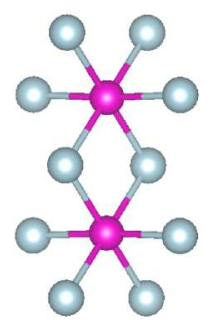

(c)

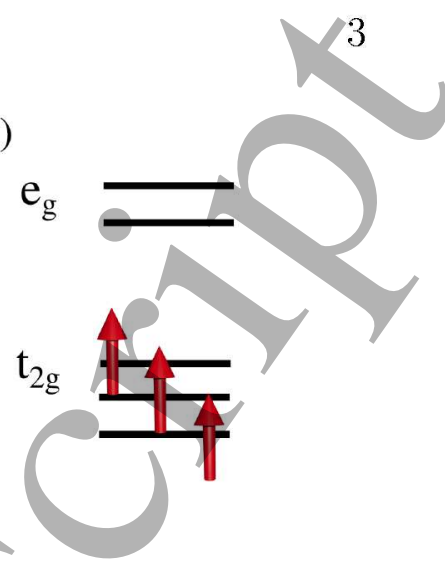

Figure 1. (a) Crystalline structure of $\mathrm{CrI}_{3}$, showing the honeycomb arrangement of the chromium atoms. Every chromium atom has an octahedral jodine environment, where different octahedra are linked by an iodine forming an angle close to 90 degrees (b). The octahedral environment splits the d levels in the eg and $t_{2 g}$ manifolds (c). First Hund rule favors the $S=3 / 2$ state, with 3 fully polarized electrons in the $t_{2 g}$ manifold.

atomic planes of I. The Cr ions are surrounded by 6 first neighbor I atoms arranged in a corner sharing octahedra. In an ionic picture, the oxidation state of $\mathrm{Cr}$ in this compound is expected to be +3 , with an electronic configuration $3 s^{0} 3 d^{3}$. In an octahedral environment the $d$ levels split into a higher energy $e_{g}$ doublet and a lower energy $t_{2 g}$ triplet.[23] Thus, we expect that $\mathrm{Cr}^{3+}$ ions in this environment have $S=3 / 2$, with 3 electrons occupying the $t_{2 g}$ manifold, complying with first Hund rule (see Fig (1)c. The lack of orbital degeneracy results in an orbital singlet[23] with a quenched orbital moment. This picture is consistent with the observed[19] saturation magnetization of bulk $\mathrm{CrI}_{3}$, that yields a magnetic moment of $\simeq 3 \mu_{B}$ per $\mathrm{Cr}$ atom, that can be explained with $S=3 / 2$ and $L=0$.

Single ion magnetic anisotropy is originated by the interplay of spin orbit coupling and the crystal field. In magnetic ions with a finite orbital moment, magnetic anisotropy scales like $\mathcal{E}_{\mathrm{MAE}} \propto \lambda\langle\vec{L}\rangle \cdot\langle\vec{S}\rangle$, where $\lambda$ is the magnetic ion atomic spin orbit coupling. However, when the orbital moment is quenched $(\langle\vec{L}\rangle=0)$, this lowest order non-zero contribution arises from quantum fluctuations of the orbital moment, and is given by $\mathcal{E}_{\mathrm{MAE}} \propto \frac{\lambda^{2}}{\Delta}$, where $\Delta$ is the energy separation with the crystal field excited states of the ion. Given that $\lambda \simeq 10 \mathrm{meV}$ for $\mathrm{Cr}$, [24] and $\Delta$ is in the range of $500 \mathrm{meV}$, single ion anisotropy energies are very often way below $1 \mathrm{meV}$. In a purely octahedral environment this quadratic contribution would actually vanish, $[23,25]$ and the magnetic anisotropy energy would scale like $\mathcal{E}_{\mathrm{MAE}} \propto \frac{\lambda^{4}}{\Delta^{3}}$, resulting in an extremely small single ion anisotropy. Based on these considerations, single ion anisotropy of $\mathrm{Cr}^{3+}$ in $\mathrm{CrI}_{3}$ should arise from the distortion of the octahedral environment.

Magnetic interactions between magnetic ions separated by non-magnetic ligands arise via the super-exchange mechanism proposed by P. W. Anderson.[26] This involves the virtual excitation of excited states where charge is transferred, during a Heisenberg time, from the ligand to the magnetic cations. This virtual processes reduce the total energy of the system and depend on the relative spin orientation of the magnetic atoms. 
On the origin of magnetic anisotropy in two dimensional $\mathrm{CrI}_{3}$
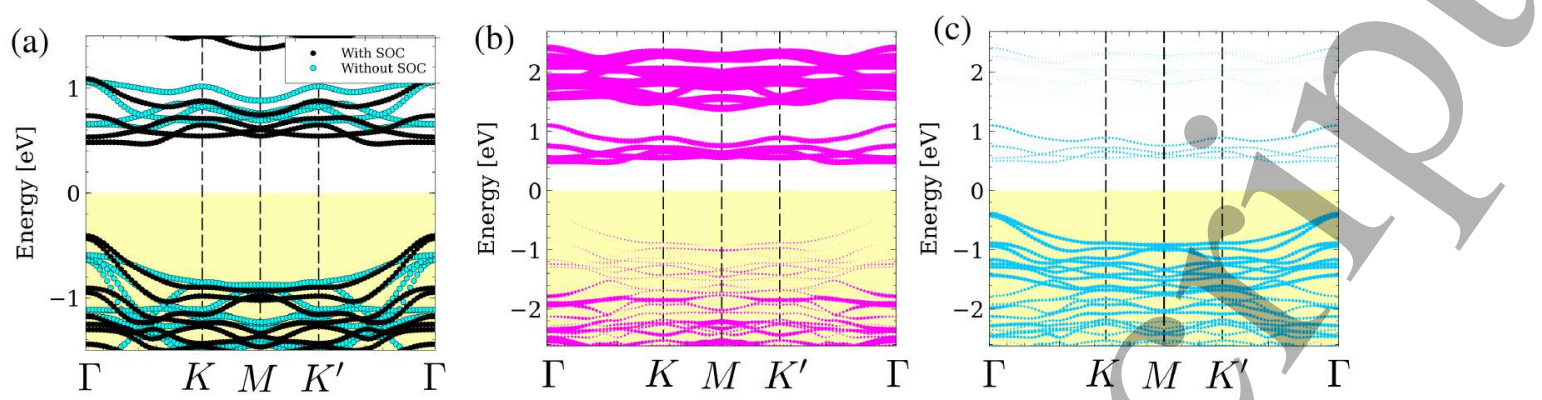

Figure 2. (a) Band structure of the system in the ferromagnetic state, without SOC (light blue) and with SOC (black). The large variation upon switching on spin orbit coupling cannot be accounted by the energy scale of the spin orbit coupling of chromium. Projection of the band structure over the chromium (b) and iodine (c) atoms. From panels $(\mathrm{b}, \mathrm{c})$ is inferred that the valence band shows a strong iodine character and that the conduction band is dominant from chromium, but with a non zero contribution from iodine. This chromium-iodine mixing is the responsible of the large variation of the conduction band upon switching on SOC, and is the ultimate responsible of the anisotropic exchange.

The sign of this exchange interaction depends both on the angle $\theta$ formed by the two chemical bonds connecting the ligand and the magnetic atoms and on the filling of the $d$ levels of the cations. A set of rules to predict the sign of the interactions was proposed, independently, by J. B. Goodenough[27] and Kanamori.[28] In particular, ferromagnetic interactions are maximal when the $\theta=90^{\circ}$. For $\mathrm{CrI}_{3}$, the angle $\theta \simeq 93^{\circ}$, which accounts[29] for the ferromagnetic interactions. As long as spin-orbit interactions are neglected, these exchange interactions are always spin rotational invariant and can be described with a Heisenberg coupling $J \vec{S}_{1} \cdot \vec{S}_{2}$.

The possibility of magnetic anisotropy in the superexchange interactions in magnetic insulators was proposed early on by T. Moriya.[30] In his seminar work, he considered the anisotropic interactions originated by spin-orbit coupling in the magnetic ions. He found two types of addition to the Heisenberg coupling. The first are the Dzyaloshinski-Moriya (DM) term or antisymmetric exchange, $\vec{D}_{i j} \cdot\left(\vec{S}_{i} \times \vec{S}_{j}\right)$, postulated by Dzyaloshinski.[31] The second is the anisotropic symmetric exchange, $\lambda S_{i}^{z} S_{j}^{z}$.

In the case of exchange mediated by an anion, the DM vector can be written as [32] $\vec{D}_{i j}=\vec{r}_{i} \times \vec{r}_{j}$, where $\vec{r}_{i}, \vec{r}_{j}$ link the anion with the two magnetic atoms. The DM favors non-collinear ground states. However, this term is absent in the $\mathrm{CrI}_{3}$ crystal, since the two paths mediated by iodine contribute to with a DM vector with opposite sign that yield a net zero contribution. In contrast, the anisotropic symmetric exchange term is allowed by symmetry and, as we show below, it is definitely important in $\mathrm{CrI}_{3}$. The symmetric and antisymmetric contributions to the anisotropic superexchange scale with $\lambda_{I}^{2}$ and $\lambda$, respectively, [30] where $\lambda_{I} \simeq 0.6 \mathrm{eV}$, is the atomic spin orbit coupling of iodine. [24] 
On the origin of magnetic anisotropy in two dimensional $\mathrm{CrI}_{3}$

\section{Density functional methods}

We perform density functional theory calculations with the pseudo-potential code Quantum Espresso[33] and the all-electron code Elk[34]. Monolayer structures were relaxed with Quantum Espresso, Projector augmented wave (PAW) pseudopotentials[35, 36] and PBE exchange correlation functional[37] in the ferromagnetic configuration. With the relaxed structures, calculation with Elk are carried out using spin orbit coupling in the non-collinear formalism, DFT $+U$ with the Yukawa scheme[38] $(J=0.7$ $\mathrm{eV}$ and $U=2.7 \mathrm{eV}$ ) in the fully localized limit and LDA exchange correlation functional.[39] We have verified that exchange energies with LDA or GGA, with or without DFT $+\mathrm{U}$ give qualitatively similar results.

The calculations of magnetic anisotropy require careful convergence of the total energy. We found that converging the total energy $10^{-8} \mathrm{eV}$ yields stable results. We have used the feature of Elk that permits to tune the overall strength of spin orbit interaction by a dimensionless constant scale factor, that we call $\alpha$. Thus, for $\alpha>1$ the size of the spin orbit coupling is increased above its actual value. In addition, we have introduced a modification in the source code of Elk in order to selectively turn on and off the spin orbit coupling in the two different atoms independently, so that we now have two dimensionless scale factors, $\alpha_{I}$ and $\alpha_{C r}$. As we discuss below, these two resources permit to to trace the origin of the magnetic anisotropy, as we discuss now.

\section{Electronic properties of $\mathrm{CrI}_{3}$}

We now describe the most salient electronic properties of $\mathrm{CrI}_{3}$, as described within our DFT calculations, in line with previous work $[20,40]$. The calculations show that $\mathrm{CrI}_{3}$ is a ferromagnetic semiconductor. The magnetic moment resides mostly in the Cr atoms, with a residual counterpolarized magnetization on the $I$ atoms. The total magnetic moment in the unit cell is $6 \mu_{B}, 3 \mu_{B}$ per $\mathrm{Cr}$ atom. Figure $2 \mathrm{a}$ shows the band structure, calculated with and without SOC. The bands undergo a rather large shift, in the range of $0.1 \mathrm{eV}$, when SOC is included,. The size of this shift is a first indication that the spin orbit interaction of iodine atoms plays an important role,[41] as spin orbit coupling in $\mathrm{Cr}$ is much smaller than $0.1 \mathrm{eV}$, Figure $2 \mathrm{~b}, \mathrm{c}$ shows the bands weighted over the projection on the $d$ orbitals of Cr (Fig. 2b) and the $p$ orbitals of I (Fig. 2c). It is apparent that the top of the valence band is formed mostly by spin unpolarized $p$ orbitals of the I atoms and the conduction band is formed by the minority spin $d$ orbitals of Cr. The majority spin $d$ orbitals, of the $t_{2 g}$ manifold, are found $2 \mathrm{eV}$ below the top of the valence bands. $\neq$ The shape of the magnetization field, not shown, clearly shows that the magnetic moment resides in orbitals with $t_{2 g}$ symmetry, in line with previous results. [40]

$\ddagger$ In the case $U=0$, the weight of the $t_{2 g}$ orbitals at the top of the valence band increases. 
On the origin of magnetic anisotropy in two dimensional $\mathrm{CrI}_{3}$

(a)

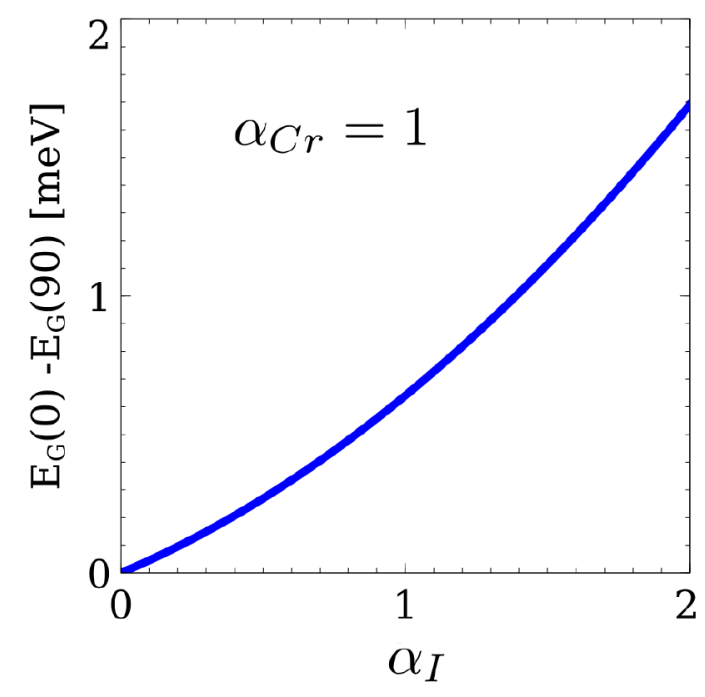

(b)

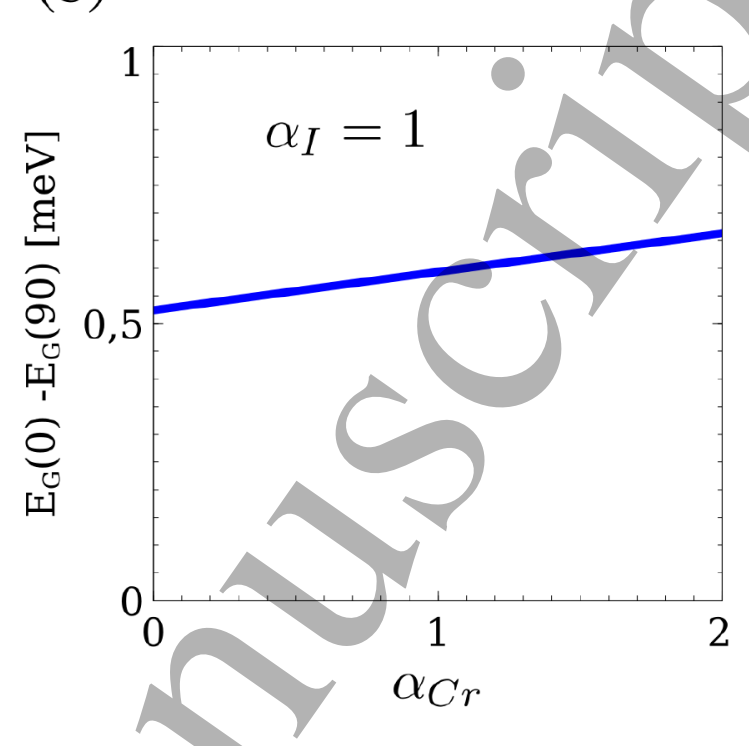

Figure 3. (a) Evolution of the magnetic anisotropy energy as a function of the spinorbit coupling in iodine $\alpha_{I}$, keeping the spin-orbit coupling in $\mathrm{Cr}$ to the real value $\alpha_{C r}=1$ (b) Same, reverting the roles of I and Cr. These curves show the dominant contribution of iodine spin orbit coupling to the MAE.

\section{Magnetic anisotropy}

We are now in position to discuss the main topic of this work, magnetic anisotropy. We have verified that the in-plane anisotropy is negligibly small. Therefore, in the following we focus on the off-plane anisotropy and we compute the quantity:

$$
\mathcal{E}_{\mathrm{MAE}}=E_{G}(0)-E_{G}(90)
$$

where $E_{G}(\theta)$ is the computed ground state energy as a function of the angle $\theta$ that forms the magnetic moment with the atomic planes. $\mathcal{E}_{\mathrm{MAE}}>0$ describes an off-plane easy axis system. For the in-plane component, we take $M_{y}=0$. In line with previous work, [20] we obtain $\mathcal{E}_{\mathrm{MAE}}=0.65 \mathrm{meV}$. Thus, the calculation predicts that the system has an easy axis anisotropy, perpendicular to the atomic planes, in agreement with the experiments.[2]

In order to study the origin of this magnetic anisotropy we compute how $\mathcal{E}_{\mathrm{MAE}}$ changes as we vary independently spin orbit coupling in two atoms. [42] To do so, here we define the DFT Hamiltonian as

$$
\mathcal{H}_{\mathrm{DFT}}\left(\alpha_{I}, \alpha_{C r}\right)=\mathcal{H}_{0}+\alpha_{I} \mathcal{H}_{I}^{S O C}+\alpha_{C r} \mathcal{H}_{C r}^{S O C}
$$

where $\mathcal{H}_{0}$ is the non relativistic Hamiltonian, $\mathcal{H}_{C r}$ the relativistic Hamiltonian correction to chromium and $\mathcal{H}_{I}$ the relativistic Hamiltonian correction to iodine. We compute magnetic anisotropy energy from Eq. 1, keeping at the default value $\alpha_{C r, I}=1$ only one of the species, and ramping the other. The results are shown in Figs. 3a,b and permit to conclude that MAE arises predominantly from the spin orbit coupling in iodine atoms. 
On the origin of magnetic anisotropy in two dimensional $\mathrm{CrI}_{3}$

This suggests that anisotropic symmetric superexchange is the likely cause of magnetic anisotropy in this compound. This also seems to indicate that the local moments do not have a strong single ion anisotropy, and therefore they are not properly described as Ising spins.

\subsection{Spin Hamiltonian}

In order to validate these hypothesis, we now propose a model Hamiltonian for the spins of the $\mathrm{Cr}$ atoms in the honeycomb lattice:

$$
\mathcal{H}=-\left(\sum_{i} D\left(S_{i}^{z}\right)^{2}+\frac{J}{2} \sum_{i, i^{\prime}} \vec{S}_{i} \cdot \vec{S}_{i^{\prime}}+\frac{\lambda}{2} \sum_{i, i^{\prime}} S_{i}^{z} S_{i^{\prime}}^{z}\right)
$$

where the sum over $i$ runs over the entire lattice of Cr atoms, and the sum over $i^{\prime}$ runs over the 3 atoms, the first neighbors of atom $i$. The first term in the Hamiltonian describes the easy axis single ion anisotropy and we choose $z$ as the offplane direction. The second term is the Heisenberg isotropic exchange and the final term is the anisotropic symmetric exchange. The sign convention is such that $J>0$ favors ferromagnetic interactions and $D>0$ favors off-plane easy axis. $\lambda=0$ would imply a completely isotropic exchange interaction.

We first treat Eq. 3 in the classical approximation, and we describe the spins $\vec{S}$ as dimensionless classical vectors of length $S$ in the sphere We write the energy of the ground state for 4 possible ground states, depicted in Fig. 4a: (I) ferromagnetic off-plane $(\mathrm{FM}, \mathrm{z}),(\mathrm{II})$ antiferromagnetic off-plane (AF,z), (III) ferromagnetic in-plane (FM,x) and (IV) antiferromagnetic in-plane $(\mathrm{AF}, \mathrm{x})$. We denote the corresponding classical ground state energies as $\mathcal{E}_{F M, z}, \mathcal{E}_{A F, z}, \mathcal{E}_{F M, x}, \mathcal{E}_{A F, x}$. The spin model allows to write the energetics of the different configurations normalized per unit cell (2 $\mathrm{Cr}$ atoms) as

$$
\begin{aligned}
& \mathcal{E}_{F M, z}=-2 S^{2} D-3 S^{2}(J+\lambda) \\
& \mathcal{E}_{A F, z}=-2 S^{2} D+3 S^{2}(J+\lambda) \\
& \mathcal{E}_{F M, x}=-3 S^{2} J \\
& \mathcal{E}_{A F, x}=+3 S^{2} J
\end{aligned}
$$

with $S=3 / 2$ for $\mathrm{CrI}_{3}$. In order to determine $J, D$ and $\lambda$, we use the ground state energies for these 4 configurations as obtained from our DFT calculations. In addition, we do this ramping the overall strength of the spin orbit coupling, $\alpha=\alpha_{C r}=\alpha_{I}$. For $\alpha=1$ we obtain $J=2.2 \mathrm{meV}$, in line with the results by Zhang et al.[20] Our results for $D$ and $\lambda$ are shown in Fig. 4b. It is apparent that the anisotropic symmetric exchange $\lambda$ is much bigger than the single ion anisotropy $D$, in particular for $\alpha=1$. The precise value of $D$ was affected by numerical noise in the regime where both $J$ and $\lambda$ already reached convergence, being always $D$ at least 30 times smaller that the anisotropic exchange $\lambda$. This yields a value of $\mathrm{D}$ negligible with respect any other exchange energy scale. Thus, we have $J>\lambda>>D$, which lead us to claim that the adequate spin model for $\mathrm{CrI}_{3}$ is the $\mathrm{XXZ}$ model with negligible single ion anisotropy. 
(a)

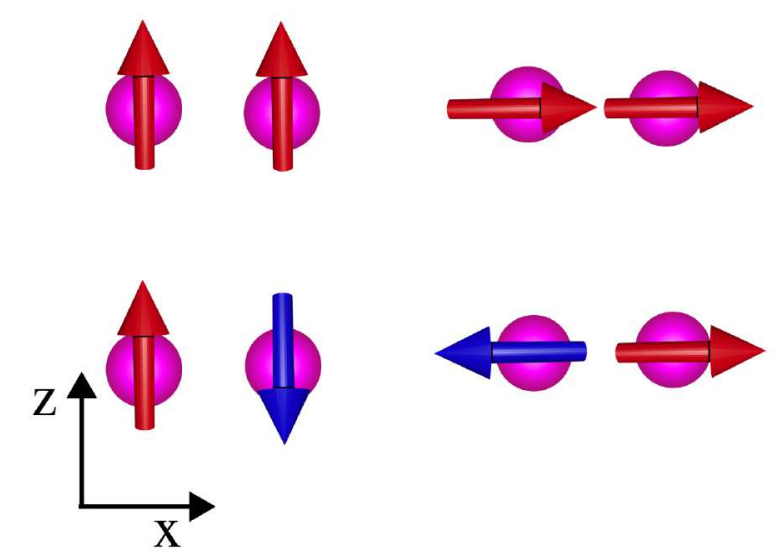

(b)

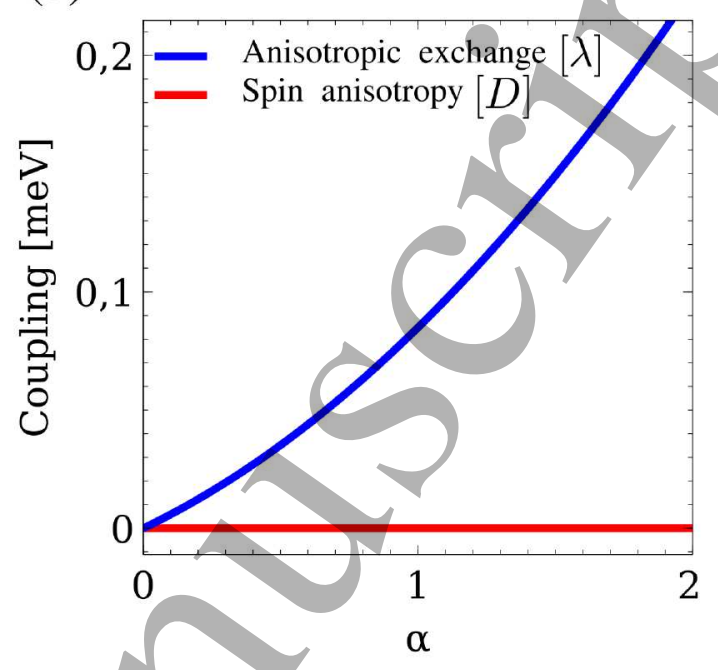

Figure 4. (a) Sketch and energetics of the different collinear magnetic configurations for two chromium atoms, showing in-plane and off-plane ferromagnetic and antiferromagnetic configurations. Comparison with DFT permits to extract $J, D$ and $\lambda$ (see text). (b) Evolution of the single ion anisotropy $D$ and anisotropic exchange $\lambda$ as a function of the spin orbit coupling $\alpha$, where $\alpha=1$ corresponds to the real value, as described at the DFT level.

This is the most important result of this work. We find $\lambda=0.04 J=0.09 \mathrm{meV}$ for $\alpha=1$. Thus, the flip-flop exchange is just 4 percent smaller than the Ising exchange $S_{i}^{z} S_{j}^{z}$, given by $J+\lambda$. Whereas the spin-flip part of exchange is actually responsible of the existence of dispersive spin wave excitations, the anisotropic term $\lambda$ opens up a gap in their spectrum, as we show below. This actually controls the transition from the ferromagnetic to the non-magnetic phase as the material is heated above $T_{c}$ :

\section{Spin Wave theory}

We now go beyond the classical approximation used in the previous section. To do that, we now treat the spins in Hamiltonian (3) as quantum mechanical $S=3 / 2$ operators. We treat the Hamiltonian within the linear spin wave approximation. To do so, we use the so called Holstein-Primakoff representation [43] of the spin operators in terms of bosonic operators

$$
\begin{aligned}
& S_{i}^{+}=\sqrt{2 S} \sqrt{1-\frac{b_{i}^{\dagger} b_{i}}{2 S} b_{i}} \\
& S_{i}^{-}=\sqrt{2 S} b_{i}^{\dagger} \sqrt{1-\frac{b_{i}^{\dagger} b_{i}}{2 S}} \\
& S_{i}^{z}=S-b_{i}^{\dagger} b_{i}
\end{aligned}
$$

with $b_{i}$ and $b_{i}^{\dagger}$ the bosonic annihilation and creation operator in site. The representation of the spin Hamiltonian (3) in terms of this bosonic operators leads a complicated non- 
linear Hamiltonian. The spin wave approximation consist in keeping only the quadratic terms in the bosonic operators $b$. This approximation is valid for a small occupation of the bosonic modes, ie, when the magnetization is closed to $S_{z} \simeq S$, ie, for small temperatures. In the spin wave approximation, the effective Hamiltonian for the spin excitations reads:

$$
\mathcal{H}_{\text {spin waves }}=\sum_{i}(2 D S+3 S(J+\lambda)) b_{i}^{\dagger} b_{i}-J S \sum_{\langle i j\rangle} b_{i}^{\dagger} b_{j}
$$

where the sum over $i$ runs over the entire lattice and the sum over $j$ runs over the first neighbors of $j$. This Hamiltonian describes bosonic excitations moving in a honeycomb lattice, with an on-site energy $\epsilon_{0}=2 D S+3 S(J+\lambda)$ and a hopping energy $J S$. Thus, the Bloch Hamiltonian for the honeycomb lattice reads

$$
\mathcal{H}_{S W}(\vec{k})=\left(\begin{array}{cc}
\epsilon_{0} & -J S f(\vec{k}) \\
-J S f^{*}(\vec{k}) & \epsilon_{0}
\end{array}\right)
$$

where $\epsilon_{0}=3 J S+2 S D+3 S \lambda, f(\vec{k})=1+e^{i \vec{k} \cdot \hat{a}_{1}}+e^{i \vec{k} \cdot \hat{a}_{2}}$ is the usual form factor for the honeycomb lattice, and $\hat{a}_{1,2}$ are the unit vectors of the triangular lattice. The resulting energy spectrum is

$$
E^{ \pm}(\vec{k})=\epsilon_{0} \pm J S \sqrt{\mid f\left(\left.\vec{k}\right|^{2}\right.}
$$

We can expand the lower band around its minima at the $\Gamma$ point, to get

$$
E^{-}(\vec{k}) \simeq \Delta_{0}+\rho k^{2}
$$

where the spin wave gap is given by

$$
\Delta_{0}=2 D S+3 S \lambda
$$

For $\mathrm{CrI}_{3}$ we can take $D=0$ and we have a spin wave gap $\Delta_{0}=3 S \lambda=0.4 \mathrm{meV}$. The so called spin stiffness is given by

$$
\rho=\frac{1}{4} J S
$$

that yields for $\mathrm{CrI}_{3}$ a value $\rho=0.82 \mathrm{meV}$. The ratio $\frac{\Delta_{0}}{\rho}=\frac{12 \lambda}{J} \simeq 0.49$ plays an important role in the following.

From Eqs. 14,15 it is apparent that if the two terms that break spin rotational invariance in the original Hamiltonian (3), $D$ and $\lambda$, vanish, the spin wave spectrum becomes gapless. Therefore, in the spin wave spectrum, both the anisotropic exchange and the single ion anisotropy create a gap in the spin waves (see Fig. 5a), so that their effect on the spin wave dispersion is similar. This implies that simple inspection of the spin wave dispersion does not provide enough information to asses whether if the correct model for a compound is single ion anisotropy or anisotropic exchange, and input from a microscopic first principles calculation is necessary. As we discuss now, the presence of their induced gap is essential to have magnetization at finite temperature. 
On the origin of magnetic anisotropy in two dimensional $\mathrm{CrI}_{3}$
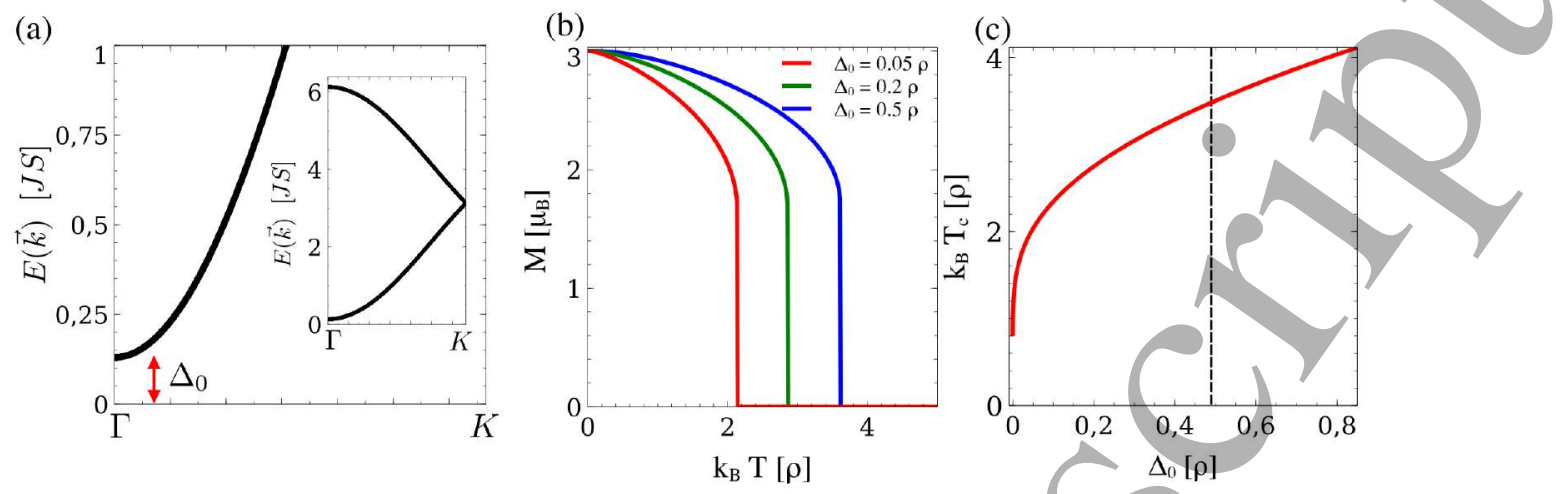

Figure 5. (a) Example of the spin wave dispersion, showing a gap in the spin excitations. (b) Selfconsistent solution of the magnetization derived with Eq. 25, showing a depleted magnetization with increasing temperature. (c) Numerical solution for the critical temperature as a function of the spin wave gap $\Delta_{0}$, showing a logarithmic dependence on $\Delta_{0}$. The dashed line in (c) is the result obtained for $\mathrm{CrI}_{3}$ with the DFT calculations.

\subsection{Low temperature magnetization}

Every magnon carries one unit of angular momentum. Therefore, in the linear spin wave framework, we can approximate the magnetization by

$$
M(T)=S-\delta M=S-\frac{1}{2(2 \pi)^{2}} \int_{B Z} \frac{d^{2} \vec{k}}{e^{\beta E(\vec{k})}-1}
$$

where $M(T)$ is the magnetization in units of $\hbar$ per $\mathrm{Cr}$ atom as a function of the temperature, the factor $1 / 2$ comes from having two $\mathrm{Cr}$ atoms in the unit cell and $\beta^{-1}=k_{B} T$. Linear spin wave theory works well when the magnon density is small. In the following we use the fact that this integral is controlled by the low energy magnons, and we approximate $E(\vec{k})$ by Eq. (14). In addition, we replace the integral over the Brillouin zone by the integral over a circle of radius $k_{c}$, chosen so that the density of magnons is properly normalized $\frac{1}{2 \pi} \int_{0}^{k_{c}} k d k=2$. Choosing this normalization includes the contribution from the high energy magnon branch in the high energy part of the dispersion. We first focus in the case $\Delta_{0}=0$, i.e. in the absence of anisotropic exchange, in that case the correction of the magnetization goes as

$$
\delta M=\frac{1}{4 \pi} \int_{0}^{k_{c}} \frac{k d k}{\beta \rho k^{2}} \rightarrow \infty
$$

This divergence signals the absence of order at finite temperature in the Heisenberg model in the gapless regime $\Delta_{0}=0$, consistent with the Mermin-Wagner theorem.[14] Therefore, the anisotropy gap is essential to protect the long range order in $2 \mathrm{D}$.

We will move now to the case of finite spin wave gap $\Delta_{0} \neq 0$. We now consider the very low temperature case, $\beta \Delta_{0}>>1$. We can then approximate[44]

$$
M(T)=M(T=0)+\delta M=S-\frac{k_{B} T}{2 \pi J S} e^{-\Delta_{0} / k_{B} T}
$$


On the origin of magnetic anisotropy in two dimensional $\mathrm{CrI}_{3}$

Thus, we expect that the magnetization will have a very weak temperature dependence for temperatures smaller than spin wave gap. According to our calculations $\Delta_{0}=0.4$ $\mathrm{meV}$, so $M(T)$ be almost maximal up to $T=5 \mathrm{~K}$.

\subsection{Estimate of $T_{c}$}

We now provide a rough estimate of the Curie temperature, based on non linear spin wave theory. We use the initial expression for spin operators, and expand them retaining the up to fourth order in the bosonic operators

$$
\begin{aligned}
& S_{i}^{+} \approx \sqrt{2 S}\left(1-\frac{b_{i}^{\dagger} b_{i}}{4 S}\right) b_{i} \\
& S_{i}^{-} \approx \sqrt{2 S} b_{i}^{\dagger}\left(1-\frac{b_{i}^{\dagger} b_{i}}{4 S}\right) \\
& S_{i}^{z}=S-b_{i}^{\dagger} b_{i}
\end{aligned}
$$

At intermediate temperatures, there is a finite number of spin waves, that is accounted by the higher order terms in bosonic operators when substituting the previous expansion in the spin Hamiltonian. In that situation, the spin Hamiltonian contains four field operators and therefore is not exactly solvable, Thus, the effect of the spin wave population is described using a mean field approximation in the spin wave Hamiltonian by means of the substitution $b_{i}^{\dagger} b_{i} b_{j}^{\dagger} b_{j} \approx\left\langle b_{i}^{\dagger} b_{i}\right\rangle b_{j}^{\dagger} b_{j}+b_{i}^{\dagger} b_{i}\left\langle b_{j}^{\dagger} b_{j}\right\rangle+\mathcal{C}$. With the previous approximation it is straightforward to check that a finite population of spin waves is equivalent to a renormalization of the hopping energy and spin wave gap as[45]

$$
\begin{aligned}
& J S \rightarrow J\left(S-\left\langle b^{\dagger} b\right\rangle\right)=J M(T) \\
& \lambda S \rightarrow \lambda\left(S-\left\langle b^{\dagger} b\right\rangle\right)=\lambda M(T)
\end{aligned}
$$

The previous substitutions lead to a selfconsistent equation for the magnetization as

$$
M=S-\frac{1}{2(2 \pi)^{2}} \int_{B Z} \frac{d^{2} \vec{k}}{e^{\beta M E(\vec{k}) / S}-1}
$$

where the integral extends over the first Brillouin zone. A qualitative behavior of the previous integral can be obtained approximating $E(\vec{k})=\Delta_{0}+\rho k^{2}$ and $e^{\beta \frac{M}{S} E(k)}-1 \approx$ $\beta M\left(\Delta_{0}+\rho k^{2}\right) / S$. As Eq. (25) has no solution for $M=0$, we define $T_{c}$ as the temperature at which the magnetization is depleted to $M=S / 2$. This leads to the following equation:

$$
k_{B} T_{c} \simeq \frac{2 \pi \rho S}{\log \frac{\Delta_{0}+8 \pi \rho}{\Delta_{0}}}=\frac{\pi J S^{2}}{2 \log \frac{\Delta_{0}+2 \pi J S}{\Delta_{0}}}
$$

A very similar result can be obtained using different spin representations.[46, 47] Equation (26), together with the numerical solution[48] of Eq. (25) in Fig(5)b, show several important results. First, $T_{c}$ is an increasing function of the spin wave gap $\Delta_{0}$ (see Fig. (5c). This is in line with the experimental results recently reported for $\mathrm{Cr}_{2} \mathrm{Ge}_{2} \mathrm{Te}_{6},[1]$ for which the major contribution to the spin wave gap comes from the 
Zeeman contribution, due to the very tiny intrinsic anisotropy, resulting in dramatic variations of $T_{c}$ as a function of the applied field. This is a feature specific of two dimensional magnets with dispersive spin waves. Second, $T_{c}$ is significantly smaller than the prediction coming from the Ising model. The exact solution for the Ising model in the honeycomb lattice[49] yields $k_{B} T_{c}=1.51 j$, where $j$ is the coupling between classical spins with $S=1$. Using this result for $\mathrm{CrI}_{3}$, we would have $k_{B} T_{c}=1.51(J+\lambda) S^{2}=85$ Kelvin, that overshoots the experimental value $45 \mathrm{~K}$.

On the other hand, using the prediction of $T_{c}$ obtained by the numerical solution of Eq. (25) shown in Fig. $5 \mathrm{c}$, we obtain a value of $k_{B} T_{c}=3.5 \rho$, for $\Delta_{0}=0.49 \rho$, that gives $T_{c}=33 \mathrm{~K}$, underestimating the the experimental value[2] $T_{c}=45 \mathrm{~K}$ by $20 \%$. Including the effect of the finite magnetic field would increase $\Delta_{0}$, and push the prediction upward. Inclusion of longer range coupling $[50,51,52,53]$ is also-expected to increase the spin stiffness, yielding a larger estimate of the critical temperature. Furthermore, a more accurate treatment must consider the explicit spin wave density of states and a more careful treatment of fluctuations close to the critical point. The discrepancy highlights the limitations of the non-linear spin wave theory, and perhaps, also those of the DFT scheme to determine the energy scales of the Hamiltonian. Nevertheless, apart from the previous limitations, our approach highlights the role played by anisotropic exchange, as the ultimate mechanism responsible to controlling the divergence in Eq. 26.

\section{Conclusions}

We have studied the origin of magnetic anisotropy in two dimensional $\mathrm{CrI}_{3}$, a recently discovered ferromagnetic two dimensional crystal with off-plane anisotropy. We have found that magnetic anisotropy in this system comes predominantly from the superexchange interaction, that gives rise to an anisotropic contribution to the conventional exchange interaction. The strength of the non Heisenberg correction is found to be controlled by the spin orbit coupling of the intermediate iodine atom. The single ion anisotropy of the magnetic $\mathrm{Cr}$ atoms is found to give a negligible contribution to magnetic anisotropy. The suppression of the single ion anisotropy due to the octahedral environment, together with large spin orbit coupling of iodine, make the anisotropic exchange the leading mechanism stabilizing the magnetic ordering in 2D $\mathrm{CrI}_{3}$. Our calculations permit to conclude that the effective spin Hamiltonian for $\mathrm{CrI}_{3}$ is a XXZ model. In turn, this implies that gapped spin waves are the essential elementary excitations that control the finite temperature properties of this new type of magnetic system. Given that spin waves in two dimensions are interesting on its own right, as they can exhibit thermal Hall effect and have topologically nontrivial phases. $[54,55,56,57,58,59]$ As an example, one can consider inducing a Dzyaloshinskii-Moriya term in a $\mathrm{CrI}_{3}$ monolayer by applying a perpendicular electric field, opening the possibility of a skyrmionic ground state whose magnonic Hamiltonian is topologically non-trivial and shows gapless edge magnonic excitations.[56] Another interesting playground would be the possibility of applying non uniform strain to the 
ferromagnetic monolayer, modulating the exchange constants and creating an artificial gauge field in the magnonic Hamiltonian.[60, 61] Therefore, the discovery of magnetic $2 \mathrm{D}$ crystals paves the way towards the exploration of these exciting phenomena.

\section{Acknowledgments}

We acknowledge F. Rivadulla for fruitful conversations. We acknowledge financial support by Marie-Curie-ITN 607904-SPINOGRAPH. JFR acknowledges financial supported by MEC-Spain (MAT2016-78625-C2). This work has been supported in part by ERDF funds through the Portuguese Operational Program for Competitiveness and Internationalization COMPETE 2020, and National Funds through FCT- The Portuguese Foundation for Science and Technology, under the project PTDC/FISNAN/4662/2014 (016656). J. L. Lado thanks the hospitality of the Departamento de Fisica Aplicada at the Universidad de Alicante.

\section{References}

[1] Cheng Gong, Lin Li, Zhenglu Li, Huiwen Ji, Alex Stern, Yang Xia, Ting Cao, Wei Bao, Chenzhe Wang, Yuan Wang, et al. Discovery of intrinsic ferromagnetism in $2 \mathrm{~d}$ van der waals crystals. arXiv preprint arXiv:1703.05753, 2017.

[2] Bevin Huang, Genevieve Clark, Efren Navarro-Moratalla, Dahlia R Klein, Ran Cheng, Kyle L Seyler, Ding Zhong, Emma Schmidgall, Michael A McGuire, David H Cobden, et al. Layerdependent ferromagnetism in a van der waals crystal down to the monolayer limit. arXiv preprint arXiv:1703.05892, 2017.

[3] Xingzhi Wang, Kezhao Du, Yu Yang Fredrik Liu, Peng Hu, Jun Zhang, Qing Zhang, Man Hon Samuel Owen, Xin Lu, Chee Kwan Gan, Pinaki Sengupta, et al. Raman spectroscopy of atomically thin two-dimensional magnetic iron phosphorus trisulfide (feps3) crystals. $2 D$ Materials, 3(3):031009, 2016.

[4] Jae-Ung Lee, Sungmin Lee, Ji Hoon Ryoo, Soonmin Kang, Tae Yun Kim, Pilkwang Kim, CheolHwan Park, Je-Geun Park, and Hyeonsik Cheong. Ising-type magnetic ordering in atomically thin feps3. Nano Letters, 16(12):7433-7438, 2016.

[5] JM Lu, O Zheliuk, Inge Leermakers, Noah FQ Yuan, Uli Zeitler, Kam Tuen Law, and JT Ye. Evidence for two-dimensional ising superconductivity in gated mos2. Science, 350(6266):1353$1357,2015$.

[6] Miguel M Ugeda, Aaron J Bradley, Yi Zhang, Seita Onishi, Yi Chen, Wei Ruan, Claudia OjedaAristizabal, Hyejin Ryu, Mark T Edmonds, Hsin-Zon Tsai, et al. Characterization of collective ground states in single-layer nbse2. Nature Physics, 12(1):92-97, 2016.

[7] Xiaoxiang Xi, Liang Zhao, Zefang Wang, Helmuth Berger, László Forró, Jie Shan, and Kin Fai Mak. Strongly enhanced charge-density-wave order in monolayer nbse2. Nature nanotechnology, 10(9):765-769, 2015.

[8] Kai Chang, Junwei Liu, Haicheng Lin, Na Wang, Kun Zhao, Anmin Zhang, Feng Jin, Yong Zhong, Xiaopeng $\mathrm{Hu}$, Wenhui Duan, et al. Discovery of robust in-plane ferroelectricity in atomic-thick snte. Science, 353(6296):274-278, 2016. 
[9] Yandong Ma, Ying Dai, Meng Guo, Chengwang Niu, Yingtao Zhu, and Baibiao Huang. Evidence of the existence of magnetism in pristine vx2 monolayers $(\mathrm{x}=\mathrm{s}, \mathrm{se})$ and their strain-induced tunable magnetic properties. ACS nano, 6(2):1695-1701, 2012.

[10] B. Sachs, T. O. Wehling, K. S. Novoselov, A. I. Lichtenstein, and M. I. Katsnelson. Ferromagnetic two-dimensional crystals: Single layers of $\mathrm{k}_{2} \mathrm{cuf}_{4}$. Phys. Rev. B, 88:201402, Nov 2013.

[11] Bheema Lingam Chittari, Youngju Park, Dongkyu Lee, Moonsup Han, Allan H MacDonald, Euyheon Hwang, and Jeil Jung. Electronic and magnetic properties of single-layer $\mathrm{m} \mathrm{p} \mathrm{x} 3$ metal phosphorous trichalcogenides. Physical Review B, 94(18):184428, 2016.

[12] Andre K Geim and Irina V Grigorieva. Van der waals heterostructures. Nature, 499(7459):419425, 2013.

[13] Ding Zhong, Kyle L Seyler, Xiayu Linpeng, Ran Cheng, Nikhil Sivadas, Bevin Huang, Emma Schmidgall, Takashi Taniguchi, Kenji Watanabe, Michael A McGuire, et al. Van der waals engineering of ferromagnetic semiconductor heterostructures for spin and valleytronics. arXiv preprint arXiv:1704.00841, 2017.

[14] N. D. Mermin and H. Wagner. Absence of ferromagnetism or antiferromagnetism in one- or two-dimensional isotropic heisenberg models. Phys. Rev. Lett., 17:1133-1136, Nov 1966.

[15] Lars Onsager. Crystal statistics. i. a two-dimensional model with an order-disorder transition. Phys. Rev., 65:117-149, Feb 1944.

[16] Ileana G Rau, Susanne Baumann, Stefano Rusponi, Fabio Donati, Sebastian Stepanow, Luca Gragnaniello, Jan Dreiser, Cinthia Piamonteze, Frithjof Nolting, Shruba Gangopadhyay, et al. Reaching the magnetic anisotropy limit of a 3d metal atom. Science, 344(6187):988-992, 2014.

[17] Jens Jensen and Allan R Mackintosh. Rare earth magnetism. Clarendon Oxford, 1991.

[18] JF Dillon Jr and CE Olson. Magnetization, resonance, and optical properties of the ferromagnet cri3. Journal of Applied Physics, 36(3):1259-1260, 1965.

[19] Michael A McGuire, Hemant Dixit, Valentino R Cooper, and Brian C Sales. Coupling of crystal structure and magnetism in the layered, ferromagnetic insulator cri3. Chemistry of Materials, 27(2):612-620, 2015.

[20] Wei-Bing Zhang, Qian Qu, Peng Zhu, and Chi-Hang Lam. Robust intrinsic ferromagnetism and half semiconductivity in stable two-dimensional single-layer chromium trihalides. Journal of Materials Chemistry C, 3(48):12457-12468, 2015.

[21] Hongbo Wang, Fengren Fan, Shasha Zhu, and Hua Wu. Doping enhanced ferromagnetism and induced half-metallicity in cri3 monolayer. EPL (Europhysics Letters), 114(4):47001, 2016.

[22] JF Dillon Jr and CE Olson. Magnetization, resonance, and optical properties of the ferromagnet cri3. Journal of Applied Physics, 36(3):1259-1260, 1965.

[23] Anatole Abragam and Brebis Bleaney. Electron paramagnetic resonance of transition ions. OUP Oxford, 2012.

[24] A. Kramida, Yu. Ralchenko, J. Reader, and and NIST ASD Team. NIST Atomic Spectra Database (ver. 5.3), [Online]. Available: http://physics.nist.gov/asd [2017, April 12]. National Institute of Standards and Technology, Gaithersburg, MD., 2015.

[25] Alejandro Ferrón, Fernando Delgado, and Joaquín Fernández-Rossier. Derivation of the spin hamiltonians for fe in mgo. New Journal of Physics, 17(3):033020, 2015.

[26] PW Anderson. Antiferromagnetism. theory of superexchange interaction. Physical Review, 79(2):350, 1950.

[27] John B Goodenough. An interpretation of the magnetic properties of the perovskite-type mixed crystalls la1- xsrxcoo3- $\lambda$. Journal of Physics and Chemistry of Solids, 6(2-3):287-297, 1958.

[28] Junjiro Kanamori. Superexchange interaction and symmetry properties of electron orbitals. Journal of Physics and Chemistry of Solids, 10(2):87-98, 1959.

[29] S. Fêldkemper and W. Weber. Generalized calculation of magnetic coupling constants for motthubbard insulators: Application to ferromagnetic cr compounds. Phys. Rev. B, 57:7755-7766, Apr 1998.

[30] Tôru Moriya. Anisotropic superexchange interaction and weak ferromagnetism. Phys. Rev., 
120:91-98, Oct 1960.

[31] I Dzyaloshinsky. A thermodynamic theory of weak ferromagnetism of antiferromagnetics. Journal of Physics and Chemistry of Solids, 4(4):241-255, 1958.

[32] Frederic Keffer. Moriya interaction and the problem of the spin arrangements in $\beta$ MnS. Phys. Rev., 126:896-900, May 1962.

[33] Paolo Giannozzi, Stefano Baroni, Nicola Bonini, Matteo Calandra, Roberto Car, Carlo Cavazzoni, Davide Ceresoli, Guido L Chiarotti, Matteo Cococcioni, Ismaila Dabo, Andrea Dal Corso, Stefano de Gironcoli, Stefano Fabris, Guido Fratesi, Ralph Gebauer, Uwe Gerstmann, Christos Gougoussis, Anton Kokalj, Michele Lazzeri, Layla Martin-Samos, Nicola Marzari, Francesco Mauri, Riccardo Mazzarello, Stefano Paolini, Alfredo Pasquarello, Lorenzo Paulatto, Carlo Sbraccia, Sandro Scandolo, Gabriele Sclauzero, Ari P Seitsonen, Alexander Smogunov, Paolo Umari, and Renata M Wentzcovitch. Quantum espresso: a modular and open-source software project for quantum simulations of materials. Journal of Physics: Condensed Matter, 21(39):395502 (19pp), 2009.

[34] Elk code, http://elk.sourceforge.net/.

[35] P. E. Blöchl. Projector augmented-wave method. Phys. Rev. B, 50:17953-17979, Dec 1994.

[36] E Kucukbenli, M Monni, BI Adetunji, X Ge, GA Adebayo, N Marzari, S De Gironcoli, and A Dal Corso. Projector augmented-wave and all-electron calculations across the periodic table: a comparison of structural and energetic properties. arXiv preprint arXiv:1404.3015, 2014.

[37] John P. Perdew, Kieron Burke, and Matthiâs Ernzerhof. Generalized gradient approximation made simple. Phys. Rev. Lett., 77:3865-3868, Oct 1996.

[38] Fredrik Bultmark, Francesco Cricchio, Oscar Grånäs, and Lars Nordström. Multipole decomposition of lda+u energy and its application to actinide compounds. Phys. Rev. B, 80:035121, Jul 2009.

[39] J. P. Perdew and Alex Zunger. Self-interaction correction to density-functional approximations for many-electron systems. Phys. Rev. B, 23:5048-5079, May 1981.

[40] Junyi Liu, Qiang Sun, Yoshiyuki Kawazoe, and Puru Jena. Exfoliating biocompatible ferromagnetic cr-trihalide monolayers. Physical Chemistry Chemical Physics, 18(13):8777-8784, 2016.

[41] Xianxin Wu, Yingxiang Cai, Qing Xie, Hongming Weng, Heng Fan, and Jiangping Hu. Magnetic ordering and multiferroicity in mni 2 . Phys. Rev. B, 86:134413, Oct 2012.

[42] K. Kośmider, J. W. González, and J. Fernández-Rossier. Large spin splitting in the conduction band of transition metal dichalcogenide monolayers. Phys. Rev. B, 88:245436, Dec 2013.

[43] $\mathrm{T}$ Holstein and $\mathrm{Hl}$ Primakoff. Field dependence of the intrinsic domain magnetization of a ferromagnet. Physical Review, 58(12):1098, 1940.

[44] Patrick Bruno. Spin-wave theory of two-dimensional ferromagnets in the presence of dipolar interactions and magnetocrystalline anisotropy. Phys. Rev. B, 43:6015-6021, Mar 1991.

[45] Daniel Stanek, Oleg P. Sushkov, and Götz S. Uhrig. Self-consistent spin-wave theory for a frustrated heisenberg model with biquadratic exchange in the columnar phase and its application to iron pnictides. Phys, Rev. B, 84:064505, Aug 2011.

[46] V Yu Irkhin, AA Katanin, and MI Katsnelson. Self-consistent spin-wave theory of layered heisenberg magnets. Physical Review B, 60(2):1082, 1999.

[47] Alexei Grechnev, Valentin Yu Irkhin, Mikhail I Katsnelson, and Olle Eriksson. Thermodynamics of a two-dimensional heisenberg ferromagnet with dipolar interaction. Physical Review B, 71(2):024427, 2005.

[48] Micheline Bloch. Magnon renormalization in ferromagnets near the curie point. Phys. Rev. Lett., 9:286-287, Oct 1962.

[49] Peter Meyer. Computational studies of pure and dilute spin models. School of Mathematics and Computing, University of Derby, 2000.

[50] Nikhil Sivadas, Matthew W. Daniels, Robert H. Swendsen, Satoshi Okamoto, and Di Xiao. Magnetic ground state of semiconducting transition-metal trichalcogenide monolayers. Phys. 
Rev. B, 91:235425, Jun 2015.

[51] Kateryna Foyevtsova, Ingo Opahle, Yu-Zhong Zhang, Harald O. Jeschke, and Roser Valentí. Determination of effective microscopic models for the frustrated antiferromagnets $\mathrm{CS}_{2} \mathrm{Cucl}_{4}$ and $\mathrm{Cs}_{2} \mathrm{Cubr}_{4}$ by density functional methods. Phys. Rev. B, 83:125126, Mar 2011.

[52] Harald O. Jeschke, Francesc Salvat-Pujol, and Roser Valentí. First-principles determination of heisenberg hamiltonian parameters for the spin- $\frac{1}{2}$ kagome antiferromagnet $\mathrm{zncu}_{3}(\mathrm{oh})_{6} \mathrm{cl}_{2}$. Phys. Rev. B, 88:075106, Aug 2013.

[53] Kateryna Foyevtsova, Harald O. Jeschke, I. I. Mazin, D. I. Khomskii, and Roser Valentí, Ab initio analysis of the tight-binding parameters and magnetic interactions in na2iro3. Phys. Rev. B, 88:035107, Jul 2013.

[54] Max Hirschberger, Robin Chisnell, Young S. Lee, and N. P. Ong. Thermal hall effect of spin excitations in a kagome magnet. Phys. Rev. Lett., 115:106603, Sep 2015.

[55] R. Chisnell, J. S. Helton, D. E. Freedman, D. K. Singh, R. I. Bewley, D. G. Nocera, and Y. S. Lee. Topological magnon bands in a kagome lattice ferromagnet. Phys. Rev. Lett., 115:147201, Sep 2015.

[56] A Roldán-Molina, AS Nunez, and Joaquín Fernández-Rossier. Topological spin waves in the atomic-scale magnetic skyrmion crystal. New Journal of Physics, 18(4):045015, 2016.

[57] SA Owerre. Topological honeycomb magnon hall effect: A calculation of thermal hall conductivity of magnetic spin excitations. Journal of Applied Physics, 120(4):043903, 2016.

[58] Vladimir A. Zyuzin and Alexey A. Kovalev. Magnon spin nernst effect in antiferromagnets. Phys. Rev. Lett., 117:217203, Nov 2016.

[59] Ran Cheng, Satoshi Okamoto, and Di Xiao. Spin nernst effect of magnons in collinear antiferromagnets. Phys. Rev. Lett., 117:217202, Nov 2016.

[60] Yaacov E Kraus and Oded Zilberberg. Quasiperiodicity and topology transcend dimensions. Nature Physics, 12(7):624-626, 2016.

[61] Yaacov E. Kraus, Zohar Ringel, and Oded Zilberberg. Four-dimensional quantum hall effect in a two-dimensional quasicrystal. Phys. Rev. Lett., 111:226401, Nov 2013. 